\title{
GENERALIZED VECTOR QUASI-EQUILIBRIUM PROBLEMS WITH SET-VALUED MAPPINGS
}

\author{
JIAN WEN PENG AND DAO LI ZHU
}

Received 26 October 2005; Revised 5 March 2006; Accepted 12 April 2006

A new mathematical model of generalized vector quasiequilibrium problem with setvalued mappings is introduced, and several existence results of a solution for the generalized vector quasiequilibrium problem with and without $\Phi$-condensing mapping are shown. The results in this paper extend and unify those results in the literature.

Copyright (C) 2006 J. W. Peng and D. L. Zhu. This is an open access article distributed under the Creative Commons Attribution License, which permits unrestricted use, distribution, and reproduction in any medium, provided the original work is properly cited.

\section{Introduction}

Throughout this paper, let $Z, E$, and $F$ be topological vector spaces, let $X \subseteq E$ and $Y \subseteq F$ be nonempty, closed, and convex subsets. Let $D: X \rightarrow 2^{X}, T: X \rightarrow 2^{Y}$ and $\Psi: X \times Y \times$ $X \rightarrow 2^{Z}$ be set-valued mappings, and let $C: X \rightarrow 2^{Z}$ be a set-valued mapping such that $C(x)$ is a closed pointed and convex cone with int $C(x) \neq \varnothing$ for each $x \in X$, where int $C(x)$ denotes the interior of the set $C(x)$. Then the generalized vector quasi-equilibrium problem with set-valued mappings (GVQEP) is to find $(\bar{x}, \bar{y})$ in $X \times Y$ such that

$$
\bar{x} \in D(\bar{x}), \quad \bar{y} \in T(\bar{x}), \quad \Psi(\bar{x}, \bar{y}, z) \nsubseteq-\operatorname{int} C(\bar{x}), \quad \forall z \in D(\bar{x})
$$

The GVQEP is a new, interesting, meaningful, and general mathematical model, which contains many mathematical models as special cases, for some examples, we have the following.

(i) If $\Psi$ is replaced by a single-valued function $f: X \times Y \times X \rightarrow Z$ and $C(x)=C$ for all $x \in X$, then the GVQEP reduces to finding $(\bar{x}, \bar{y})$ in $X \times Y$ such that

$$
\bar{x} \in D(\bar{x}), \quad \bar{y} \in T(\bar{x}), \quad f(\bar{x}, \bar{y}, z) \notin-\operatorname{int} C, \quad \forall z \in D(\bar{x}) .
$$

It was investigated by Chiang et al. in [7].

Hindawi Publishing Corporation Journal of Inequalities and Applications Volume 2006, Article ID 69252, Pages 1-12 DOI 10.1155/JIA/2006/69252 
2 Generalized vector quasi-equilibrium problem

If $\Psi$ is replaced by a scalar function $f: X \times Y \times X \rightarrow R$ and $C(x)=\{r \in R: r \geq 0\}$ for all $x \in X$, then the GVQEP reduces to finding $(\bar{x}, \bar{y})$ in $X \times Y$ such that

$$
\bar{x} \in D(\bar{x}), \quad \bar{y} \in T(\bar{x}), \quad f(\bar{x}, \bar{y}, z) \geq 0, \quad \forall z \in D(\bar{x}) .
$$

This was investigated in $[5,6,12,13,19]$ and contains the generalized quasi-variational inequality in $[4,8,20,21]$ as a special case.

(ii) If $D(x)=X$ for all $x \in X$ and $f=-\Psi$, then the GVQEP reduces to finding $(\bar{x}, \bar{y})$ in $X \times Y$ such that

$$
\bar{x} \in X, \quad \bar{y} \in T(\bar{x}), \quad f(\bar{x}, \bar{y}, z) \nsubseteq \operatorname{int} C(\bar{x}), \quad \forall z \in X .
$$

It is the model of GVEP3 by Fu and Wan in [10]. Fu and Wan also introduce another kind of general vector equilibrium problem (i.e., GVEP1 in [10]) which is to find $\bar{x}$ in $X$ such that for all $z \in X, \exists \bar{y} \in T(\bar{x}), f(\bar{x}, \bar{y}, z) \nsubseteq$ int $C(\bar{x})$.

(iii) If $Y=\{\bar{y}\}$ and $T(x)=\{\bar{y}\}$ for all $x \in X$, define a function $\varphi: X \times X \rightarrow 2^{Z}$ as $\varphi(x, z)=F(x, \bar{y}, z)$, then the GVQEP reduces to finding $\bar{x}$ in $X$ such that

$$
\bar{x} \in D(\bar{x}), \quad \varphi(\bar{x}, z) \nsubseteq-\operatorname{int} C(\bar{x}), \quad \forall z \in D(\bar{x}) .
$$

This was studied in $[1,16]$.

In this paper, by some maximal element theorems, we prove the existence results of a solution for the GVQEP with and without $\Phi$-condensing mappings, and we also present some existence results of a solution for the special cases of the GVQEP. The results in this paper extend and unify those results in $[1,5-7,10,12,13,15,19]$ and the references therein.

\section{Preliminaries}

In this section, we recall some definitions and some well-known results we need.

Definition 2.1 (see [2]). Let $C: X \rightarrow 2^{Z}$ be a set-valued mapping with int $C(x) \neq \varnothing$ for all $x \in X$. Let $\varphi: X \times X \rightarrow 2^{Z}$ be a set-valued mapping. Then $\varphi(x, z)$ is said to be $C_{x^{-}}$ quasiconvex-like if for all $x \in X, y_{1}, y_{2} \in X$, and $\alpha \in[0,1]$, either

$$
\varphi\left(x, \alpha y_{1}+(1-\alpha) y_{2}\right) \subseteq \varphi\left(x, y_{1}\right)-C(x)
$$

or

$$
\varphi\left(x, \alpha y_{1}+(1-\alpha) y_{2}\right) \subseteq \varphi\left(x, y_{2}\right)-C(x)
$$

Definition 2.2 (see [15]). Let $C: X \rightarrow 2^{Z}$ be a set-valued mapping such that $C(x)$ is a closed pointed and convex cone with int $C(x) \neq \varnothing$ for each $x \in X$. Then the set-valued mapping $\varphi: X \times X \rightarrow 2^{Z}$ is called to be $C_{x}$-0-diagonally quasiconvex if for any finite set 
$\left\{z_{1}, z_{2}, \ldots, z_{n}\right\}$ in $X$, and for all $x \in X$ with $x \in \operatorname{Co}\left\{z_{1}, z_{2}, \ldots, z_{n}\right\}$, there exists some $j \in$ $\{1,2, \ldots, n\}$ such that $\varphi\left(x, z_{j}\right) \nsubseteq-\operatorname{int} C(x)$.

Remark 2.3. (i) It is clear that if $Z=R$ and $C(x)=\{r \in R: r \geq 0\}$ for all $x \in X$, and $\varphi$ is a single-valued function, then the $C(x)$-0-diagonal quasiconvexity of $\varphi$ reduces to the 0 -diagonal quasiconvexity in $[22,23]$, here $\gamma=0$.

(ii) The following example shows that the $C_{x}$-0-diagonal quasiconvexity of a set-valued map $F$ is a true generalization of $C_{x}$-convex-likeness of the same $F$.

Let $E$ be a real normed space with dual space $E^{*}, X \subset E, Z=R$. Let $\|\bullet\|$ denote the norm on $E$. Let $C: X \rightarrow 2^{Z}$ be defined as $C(x)=[\|x\|,+\infty)$, for all $x \in X$, and let $\left[e_{1}, e_{2}\right]$ denote the line segment joining $e_{1}$ and $e_{2}$. Choose $p \in E^{*}$, we define a set-valued map $F: X \times X \rightarrow 2^{Z}$ as

$$
F(x, z)=\{\langle u, z-x\rangle: u \in[-2\|x\|\|z\| p,-\|x\|\|z\| p]\}, \quad \forall x \in X .
$$

Then, $F$ is $C_{x}$-0-diagonal quasiconvex in the second argument. Otherwise, there exists finite set $\left\{z_{1}, z_{2}, \ldots, z_{n}\right\} \subseteq X$, and there is $x \in X$ with $x=\sum_{j=1}^{n} \alpha_{j} z_{j}\left(\alpha_{j} \geq 0, \sum_{j=1}^{n} \alpha_{j}=1\right)$ such that for all $j=1,2, \ldots, n, F\left(x, z_{j}\right) \subseteq-\operatorname{int} C(x)$. Then for each $j$, for all $\lambda_{j} \in[0,1]$, we have

$$
\left\langle\lambda_{j}\left(-2\|x\|\left\|z_{j}\right\| p\right)+\left(1-\lambda_{j}\right)\left(-\|x\|\left\|z_{j}\right\| p\right), z_{j}-x\right\rangle<-\|x\| \leq 0 .
$$

It follows that

$$
\left\langle p, z_{j}-x\right\rangle>0, \quad j=1,2, \ldots, n
$$

Then we have

$$
0<\sum_{j=1}^{n} \alpha_{j}\left\langle p, z_{j}-x\right\rangle=\langle p, x-x\rangle=0
$$

a contradiction.

Definition 2.4 (see $[3,14]$ ). Let $E$ and $F$ be two topological spaces and let $T: E \rightarrow 2^{F}$ be a set-valued mapping.

(1) A subset $X \subseteq E$ is said to be compactly open (resp., compactly closed) in $E$ if for any nonempty compact subset $K$ of $E, X \cap K$ is open (resp., closed) in $K$.

(2) $T$ is said to be upper semicontinuous if the set $\{x \in E: T(x) \subseteq V\}$ is open in $E$ for every open subset $V$ of $F$.

(3) $T$ is said to have open (resp., compactly open) lower sections if the set $T^{-1}(y)=$ $\{x \in E: y \in T(x)\}$ is open (resp., compactly open) in $E$ for each $y \in F$.

Remark 2.5. Clearly each open (resp., closed) subset of $E$ is compactly open (resp., compactly closed), and the converse is not true in general.

Definition 2.6 (see [9]). Let $E$ be a Hausdorff topological space and $L$ a lattice with least element, denoted by 0 . A map $\Phi: 2^{E} \rightarrow L$ is a measure of noncompactness provided that the following conditions hold for all $M, N \in 2^{E}$ : 
4 Generalized vector quasi-equilibrium problem

(i) $\Phi(M)=0$ if and only if $M$ is precompact (i.e., it is relatively compact);

(ii) $\Phi(\overline{\mathrm{Co}} M)=\Phi(M)$, where $\overline{c o} M$ denotes the convex closure of $M$;

(iii) $\Phi(M \cup N)=\max \{\Phi(M), \Phi(N)\}$.

Definition 2.7 (see [9]). Let $\Phi: 2^{E} \rightarrow L$ be a measure of noncompactness on $E$ and $D \subseteq E$. A set-valued mapping $T: D \rightarrow 2^{E}$ is called $\Phi$-condensing provided that if $M \subseteq D$ with $\Phi(T(M)) \geq \Phi(M)$, then $M$ is relatively compact.

Remark 2.8. Note that every set-valued mapping defined on a compact set is $\Phi$ condensing for any measure of noncompactness $\Phi$. If $E$ is locally convex and $T: D \rightarrow 2^{E}$ is a compact set-valued mapping (i.e., $T(X)$ is precompact), then $T$ is $\Phi$-condensing for any measure of noncompactness $\Phi$. It is clear that if $T: D \rightarrow 2^{E}$ is $\Phi$-condensing and $T^{*}: D \rightarrow 2^{E}$ satisfies $T^{*}(x) \subseteq T(x)$ for all $x \in D$, then $T^{*}$ is also $\Phi$-condensing.

Let CoA denote the convex hull of the set $A$.

Lemma 2.9 (see [11]). Let $X$ be a nonempty convex subset of a Hausdorff topological vector space $E$ and let $S: X \rightarrow 2^{X}$ be a set-valued mapping such that for each $x \in X, x \notin \operatorname{Co}(S(x))$ and for each $y \in X, S^{-1}(y)$ is open in $X$. Suppose further that there exist a nonempty compact subset $N$ of $X$ and a nonempty compact convex subset $B$ of $X$ such that $\operatorname{Co}(S(x)) \cap B \neq \varnothing$ for all $x \in X \backslash N$. Then there exists a point $\bar{x} \in X$ such that $S(\bar{x})=\varnothing$.

Lemma 2.10 (see [14]). Let $X$ be a nonempty closed and convex subset of a locally convex topological vector space $E$ and let $\Phi: 2^{E} \rightarrow L$ be a measure of noncompactness on $E$. Suppose that $S: X \rightarrow 2^{X}$ is a set-valued mapping such that the following conditions are satisfied:

(i) for each $x \in X, x \notin S(x)$;

(ii) for each $y \in X, S^{-1}(y)$ is compactly open in $X$;

(iii) the set-valued mapping $S: X \rightarrow 2^{X}$ is $\Phi$-condensing. Then there exists $\bar{x} \in X$ such that $S(\bar{x})=\varnothing$.

\section{Existence results}

Some existence results of a solution for the GVQEP without $\Phi$-condensing mappings are first shown.

Theorem 3.1. Let $Z$ be a topological vector space, let $E$ and $F$ be two Hausdorff topological vector spaces, let $X \subseteq E$ and $Y \subseteq F$ be nonempty and convex subsets, let $C: X \rightarrow 2^{Z}$ be a setvalued mapping such that $C(x)$ is a closed pointed and convex cone with int $C(x) \neq \varnothing$ for each $x \in X$, let $D: X \rightarrow 2^{X}$ and $T: X \rightarrow 2^{Y}$ be set-valued mappings with nonempty convex values and open lower sections, and the set $W=\{(x, y) \in X \times Y: x \in D(x)$ and $y \in T(x)\}$ is closed in $X \times Y$. Let $\Psi: X \times Y \times X \rightarrow 2^{Z}$ be a set-valued mapping. Assume that

(i) $M=Z \backslash(-\operatorname{int} C): X \rightarrow 2^{Z}$ is upper semicontinuous;

(ii) for each $y \in Y, \Psi(x, y, z)$ is $C_{x}$-0-diagonally quasiconvex;

(iii) for all $z \in X,(x, y) \mapsto \Psi(x, y, z)$ is upper semicontinuous on $X \times Y$ with compact values;

(iv) there exist nonempty and compact subsets $N \subseteq X$ and $K \subseteq Y$ and nonempty, compact, and convex subsets $B \subseteq X, A \subseteq Y$ such that for all $(x, y) \in X \times Y \backslash N \times K \exists \bar{u} \in$ $B, \bar{v} \in A$ satisfying $\bar{u} \in D(x), \bar{v} \in T(x)$ and $\Psi(x, y, \bar{u}) \subseteq-\operatorname{int} C(x)$. 
Then, there exists $(\bar{x}, \bar{y})$ in $X \times Y$ such that

$$
\bar{x} \in D(\bar{x}), \quad \bar{y} \in T(\bar{x}), \quad \Psi(\bar{x}, \bar{y}, z) \nsubseteq-\operatorname{int} C(\bar{x}), \quad \forall z \in D(\bar{x}) .
$$

That is, the solution set of the GVQEP is nonempty.

Proof. Define a set-valued mapping $P: X \times Y \rightarrow 2^{X}$ by

$$
P(x, y)=\{z \in X: \Psi(x, y, z) \subseteq-\operatorname{int} C(x)\}, \quad \forall(x, y) \in X \times Y .
$$

It is needed to prove that $x \notin \operatorname{Co}(P(x, y))$ for all $(x, y) \in X \times Y$. To see this, suppose, by way of contradiction, that there exist some points $(\bar{x}, \bar{y}) \in X \times Y$ such that $\bar{x} \in \operatorname{Co}(P(\bar{x}, \bar{y}))$. Then there exist finite points $z_{1}, z_{2}, \ldots, z_{n}$ in $X$, and $\alpha_{j} \geq 0$ with $\sum_{j=1}^{n} \alpha_{j}=1$ such that $\bar{x}=\sum_{j=1}^{n} \alpha_{j} z_{j}$ and $z_{j} \in P(\bar{x}, \bar{y})$ for all $j=1,2, \ldots, n$. That is, $\Psi\left(\bar{x}, \bar{y}, z_{j}\right) \subseteq-\operatorname{int} C(\bar{x}), j=$ $1,2, \ldots, n$, which contradicts the fact that $\Psi(x, \bar{y}, z)$ is $C_{x}$-0-diagonal quasiconvex. Therefore, $x \notin \operatorname{Co}(P(x, y))$, for all $(x, y) \in X \times Y$. Now, it is needed to prove that the set $P^{-1}(z)=$ $\{(x, y) \in X \times Y: \Psi(x, y, z) \subseteq-\operatorname{int} C(x)\}$ is open for each $z \in X$. That is, $P$ has open lower sections on $X \times Y$. It is only needed to prove that $Q(z)=\{(x, y) \in X \times Y: \Psi(x, y, z) \nsubseteq$ $-\operatorname{int} C(x)\}$ is closed for all $z \in X$. In fact, consider a net $\left(x_{t}, y_{t}\right) \in Q(z)$ such that $\left(x_{t}, y_{t}\right) \rightarrow$ $(x, y) \in X \times Y$. Since $\left(x_{t}, y_{t}\right) \in Q(z)$, there exists $u_{t} \in \Psi\left(x_{t}, y_{t}, z\right)$ such that $u_{t} \notin-\operatorname{int} C\left(x_{t}\right)$. From the upper semicontinuity and compact values of $\Psi$ on $X \times Y$ and [17, Proposition 1], it suffices to find a subset $\left\{u_{t_{j}}\right\}$ which converges to some $u \in \Psi(x, y, z)$, where $u_{t_{j}} \in \Psi\left(x_{t_{j}}, y_{t_{j}}, z\right)$. Since $\left(x_{t_{j}}, y_{t_{j}}\right) \rightarrow(x, y)$, by [3, Proposition 7, page 110] and the upper semicontinuity of $M$, it follows that $u \notin-\operatorname{int} C(x)$, and hence $(x, y) \in Q(z), Q(z)$ is closed.

Hence, $P$ has open lower sections, and by [18, Lemma 2], we know that $\operatorname{Co} P: X \times Y \rightarrow$ $2^{X}$ also has open lower sections. Also define another set-valued mapping $S: X \times Y \rightarrow$ $2^{X \times Y}$ by

$$
S(x, y)= \begin{cases}{[D(x) \cap \operatorname{Co} P(x, y)] \times T(x)} & \text { if }(x, y) \in W \\ D(x) \times T(x) & \text { if }(x, y) \notin W\end{cases}
$$

Then, it is clear that for all $(x, y) \in X \times Y, S(x, y)$ is convex, and $(x, y) \notin \operatorname{Co}(S(x, y))=$ $S(x, y)$.

Since for all $(u, v) \in X \times Y$,

$$
\begin{aligned}
S^{-1}(u, v) & =\left[\operatorname{Co} P^{-1}(u) \cap\left(D^{-1}(u) \times Y\right) \cap\left(T^{-1}(v) \times Y\right)\right] \\
& \cup\left[(X \times Y \backslash W) \cap\left(D^{-1}(u) \times Y\right) \cap\left(T^{-1}(v) \times Y\right)\right],
\end{aligned}
$$

and $D^{-1}(u) \times Y, T^{-1}(v) \times Y, \operatorname{Co} P^{-1}(u)$, and $X \times Y \backslash W$ are open in $X \times Y$, we have $S^{-1}(u, v)$ open in $X \times Y$.

From condition (iv), there exist nonempty and compact subset $N \times K \subseteq X \times Y$ and nonempty, compact, and convex subset $B \times A \subseteq X \times Y$ such that for all $(x, y) \in X \times Y \backslash$ $N \times K, \exists(\bar{u}, \bar{v}) \in S(x, y) \cap(B \times A)$. And so $\operatorname{Co}(S(x, y)) \cap(B \times A) \neq \varnothing$. Hence, by Lemma 2.9, $\exists(\bar{x}, \bar{y}) \in X \times Y$ such that $S(\bar{x}, \bar{y})=\varnothing$. Since for all $(x, y) \in X \times X, D(x)$ and $T(y)$ are 
nonempty, we have $(\bar{x}, \bar{y}) \in W$ and $D(\bar{x}) \cap \operatorname{Co} P(\bar{x}, \bar{y})=\varnothing$. This implies that $(\bar{x}, \bar{y}) \in W$ and $D(\bar{x}) \cap P(\bar{x}, \bar{y})=\varnothing$. Therefore,

$$
\bar{x} \in D(\bar{x}), \quad \bar{y} \in T(\bar{x}), \quad \Psi(\bar{x}, \bar{y}, z) \nsubseteq-\operatorname{int} C(\bar{x}), \quad \forall z \in D(\bar{x}) .
$$

That is, the solution set of the GVQEP is nonempty. The proof is completed.

Theorem 3.2. Let $Z$ be a topological vector space, let $E$ and $F$ be two Hausdorff topological vector spaces, let $X \subseteq E$ and $Y \subseteq F$ be nonempty and convex subsets, let $C: X \rightarrow 2^{Z}$ be a setvalued mapping such that $C(x)$ is a closed pointed and convex cone with int $C(x) \neq \varnothing$ for each $x \in X$, let $D: X \rightarrow 2^{X}$ and $T: X \rightarrow 2^{Y}$ be set-valued mappings with nonempty convex values and open lower sections, and the set $W=\{(x, y) \in X \times Y: x \in D(x)$ and $y \in T(x)\}$ is closed in $X \times Y$. Let $\Psi: X \times Y \times X \rightarrow 2^{Z}$ be a set-valued mapping. Assume that

(i) $M=Z \backslash(-\operatorname{int} C): X \rightarrow 2^{Z}$ is upper semicontinuous;

(ii) for all $x \in X$, for all $y \in Y, \Psi(x, y, x) \nsubseteq-\operatorname{int} C(x)$;

(iii) for each $(x, y) \in X \times Y$, the set $P(x, y)=\{z \in X: \Psi(x, y, z) \subseteq-\operatorname{int} C(x)\}$ is a convex set;

(iv) for all $z \in X,(x, y) \mapsto \Psi(x, y, z)$ is upper semicontinuous on $X \times Y$ with compact values;

(v) there exist nonempty and compact subsets $N \subseteq X$ and $K \subseteq Y$ and nonempty, compact, and convex subsets $B \subseteq X, A \subseteq Y$ such that for all $(x, y) \in X \times Y \backslash N \times K \exists \bar{u} \in$ $B, \bar{v} \in A$ satisfying $\bar{u} \in D(x), \bar{v} \in T(x)$, and $\Psi(x, y, \bar{u}) \subseteq-\operatorname{int} C(x)$.

Then, there exists $(\bar{x}, \bar{y})$ in $X \times Y$ such that

$$
\bar{x} \in D(\bar{x}), \quad \bar{y} \in T(\bar{x}), \quad \Psi(\bar{x}, \bar{y}, z) \nsubseteq-\operatorname{int} C(\bar{x}), \quad \forall z \in D(\bar{x}) .
$$

That is, the solution set of the GVQEP is nonempty.

Proof. By Theorem 3.1, it is only needed to prove that $\Psi(x, y, z)$ is $C_{x}$-0-diagonally quasiconvex for all $y \in Y$. If not, then there exist $y \in Y$ and some finite set $\left\{z_{1}, z_{2}, \ldots, z_{n}\right\}$ in $X$, and some point $x \in X$ with $x \in \operatorname{Co}\left\{z_{1}, z_{2}, \ldots, z_{n}\right\}$, such that for each $j=1,2, \ldots, n$, $\Psi\left(x, y, z_{j}\right) \subseteq-\operatorname{int} C(x)$. Since $P(x, y)=\{z \in X: \Psi(x, y, z) \subseteq-\operatorname{int} C(x)\}$ is a convex set, $x \in P(x, y)$, that is, $\Psi(x, y, x) \subseteq-\operatorname{int} C(x)$, which contradicts the condition (ii). The proof is completed.

Then, some existence results of a solution for the GVQEP with $\Phi$-condensing mappings are shown as follows.

Theorem 3.3. Let $Z$ be a topological vector space, let $E$ and $F$ be two locally convex topological vector spaces, let $X \subseteq E$ and $Y \subseteq F$ be nonempty, closed, and convex subsets, let $C: X \rightarrow 2^{Z}$ be a set-valued mapping such that $C(x)$ is a closed pointed and convex cone with int $C(x) \neq \varnothing$ for each $x \in X, D: X \rightarrow 2^{X}$, and $T: X \rightarrow 2^{Y}$ be set-valued mappings with nonempty convex values and compactly open lower sections, and the set $W=\{(x, y) \in$ $X \times Y: x \in D(x)$ and $y \in T(x)\}$ is compactly closed in $X \times Y$. Let $\Psi: X \times Y \times X \rightarrow 2^{Z}$ be a set-valued mapping and $\Phi: 2^{E} \rightarrow L$ be a measure of noncompactness on $E$. Assume that

(i) $M=Z \backslash(-\operatorname{int} C): X \rightarrow 2^{Z}$ is upper semicontinuous on each compact subset of $X$;

(ii) for each $y \in Y, \Psi(x, y, z)$ is $C_{x}$-0-diagonally quasiconvex; 
(iii) for all $z \in X,(x, y) \mapsto \Psi(x, y, z)$ is upper semicontinuous on each compact subset of $X \times Y$ with compact values;

(iv) the set-valued map $D \times T: X \times X \rightarrow 2^{X \times Y}$ defined as $(D \times T)(x, y)=D(x) \times T(y)$, for all $(x, y) \in X \times X$, is $\Phi$-condensing.

Then, there exists $(\bar{x}, \bar{y})$ in $X \times Y$ such that

$$
\bar{x} \in D(\bar{x}), \quad \bar{y} \in T(\bar{x}), \quad \Psi(\bar{x}, \bar{y}, z) \nsubseteq-\operatorname{int} C(\bar{x}), \quad \forall z \in D(\bar{x}) .
$$

That is, the solution set of the GVQEP is nonempty.

Proof. Let $P: X \times Y \rightarrow 2^{X}$ and $S: X \times Y \rightarrow 2^{X \times Y}$ be the same as defined in the proof of Theorem 3.1. Following similar argument in the proof of Theorem 3.1, we have for all $(x, y) \in X \times Y, S(x, y)$ is convex, $(x, y) \notin S(x, y)$, and $S$ has compactly open lower sections in $X \times Y$.

By the definition of $S, S(x, y) \subseteq D(x) \times T(x)$ for all $(x, y) \in X \times Y$. Since $D \times T$ is $\Phi$ condensing, so is $S$. Hence, by Lemma 2.10, $\exists(\bar{x}, \bar{y}) \in X \times Y$ such that $S(\bar{x}, \bar{y})=\varnothing$. Since for all $(x, y) \in X \times X, D(x)$, and $T(y)$ are nonempty, we have $(\bar{x}, \bar{y}) \in W$ and $D(\bar{x}) \cap$ $\operatorname{Co} P(\bar{x}, \bar{y})=\varnothing$. This implies that $(\bar{x}, \bar{y}) \in W$ and $D(\bar{x}) \cap P(\bar{x}, \bar{y})=\varnothing$. Therefore,

$$
\bar{x} \in D(\bar{x}), \quad \bar{y} \in T(\bar{x}), \quad \Psi(\bar{x}, \bar{y}, z) \nsubseteq-\operatorname{int} C(\bar{x}), \quad \forall z \in D(\bar{x}) .
$$

That is, the solution set of the GVQEP is nonempty. The proof is completed.

By Theorem 3.3, and by similar argument to those in the proof of Theorem 3.2, it is easy to obtain the following result.

Theorem 3.4. Let $Z$ be a topological vector space, let $E$ and $F$ be two locally convex topological vector spaces, let $X \subseteq E$ and $Y \subseteq F$ be nonempty, closed, and convex subsets, let $C: X \rightarrow 2^{Z}$ be a set-valued mapping such that $C(x)$ is a closed pointed and convex cone with int $C(x) \neq \varnothing$ for each $x \in X$, let $D: X \rightarrow 2^{X}$ and $T: X \rightarrow 2^{Y}$ be set-valued mappings with nonempty convex values and compactly open lower sections, and the set $W=\{(x, y) \in$ $X \times Y: x \in D(x)$ and $y \in T(x)\}$ is compactly closed in $X \times Y$. Let $\Psi: X \times Y \times X \rightarrow 2^{Z}$ be a set-valued mapping and let $\Phi: 2^{E} \rightarrow L$ be a measure of noncompactness on E. Assume that

(i) $M=Z \backslash(-\operatorname{int} C): X \rightarrow 2^{Z}$ is upper semicontinuous on each compact subset of $X$;

(ii) for all $x \in X$, for all $y \in Y, \Psi(x, y, x) \nsubseteq-\operatorname{int} C(x)$;

(iii) for each $(x, y) \in X \times Y$, the set $P(x, y)=\{z \in X: \Psi(x, y, z) \subseteq-\operatorname{int} C(x)\}$ is a convex set;

(iv) for all $z \in X,(x, y) \mapsto \Psi(x, y, z)$ is upper semicontinuous on each compact subset of $X \times Y$ with compact values;

(v) the set-valued map $D \times T: X \times X \rightarrow 2^{X \times Y}$ defined as $(D \times T)(x, y)=D(x) \times T(y)$, for all $(x, y) \in X \times X$, is $\Phi$-condensing.

Then, there exists $(\bar{x}, \bar{y})$ in $X \times Y$ such that

$$
\bar{x} \in D(\bar{x}), \quad \bar{y} \in T(\bar{x}), \quad \Psi(\bar{x}, \bar{y}, z) \nsubseteq-\operatorname{int} C(\bar{x}), \quad \forall z \in D(\bar{x}) .
$$

That is, the solution set of the GVQEP is nonempty. 
Remark 3.5. If for each $y \in Y, \Psi(x, y, z)$ is $C_{x}$-convex-like, then the condition (iii) in both Theorems 3.2 and 3.4 holds. In fact, for any $z_{1}, z_{2} \in P(x, y)$, that is, $z_{1}, z_{2} \in X, \Psi\left(x, y, z_{1}\right) \subseteq$ $-\operatorname{int} C(x)$ and $\Psi\left(x, y, z_{2}\right) \subseteq-\operatorname{int} C(x)$. Then, for all $\lambda \in[0,1], \lambda z_{1}+(1-\lambda) z_{2} \in X$ since $X$ is convex. And since $\Psi(x, y, z)$ is $C_{x}$-quasiconvex-like for all $y \in Y$, we have either

$$
\Psi\left(x, y, \lambda z_{1}+(1-\lambda) z_{2}\right) \subseteq \Psi\left(x, y, z_{1}\right)-C(x) \subseteq-C(x)-\operatorname{int} C(x) \subseteq-\operatorname{int} C(x),
$$

or

$$
\Psi\left(x, y, \lambda z_{1}+(1-\lambda) z_{2}\right) \subseteq \Psi\left(x, y, z_{2}\right)-C(x) \subseteq-C(x)-\operatorname{int} C(x) \subseteq-\operatorname{int} C(x) .
$$

In both cases, we get $\Psi\left(x, y, \lambda z_{1}+(1-\lambda) z_{2}\right) \subseteq-\operatorname{int} C(x)$. Hence, $\lambda z_{1}+(1-\lambda) z_{2} \in P(x, y)$ for all $(x, y) \in X \times Y$, and therefore $P(x, y)$ is convex.

Remark 3.6. Theorems 3.1, 3.2, 3.3, and 3.4, respectively, generalize those results in [5$7,12,13,19]$ from scalar or vector-valued case to set-valued case with noncompact and nonmonotone conditions.

By [10, Lemma 2], we know that if $\bar{x}$ is a solution of GVEP3, then it is also is a solution of GVEP1. Fu and Wan [10] got some existence results of a solution for GVEP1. Let $f=-\Psi$ and $D(x)=X$ for all $x \in X$, by Theorems 3.1 and 3.3, respectively, we can obtain the existence results of a solution for GVEP3 as follows.

Corollary 3.7. Let $Z$ be a topological vector space, let $E$ and $F$ be two Hausdorff topological vector spaces, let $X \subseteq E$ and $Y \subseteq F$ be nonempty and convex subsets, let $C: X \rightarrow 2^{Z}$ be a set-valued mapping such that $C(x)$ is a closed pointed and convex cone with int $C(x) \neq \varnothing$ for each $x \in X$, let $T: X \rightarrow 2^{Y}$ be a set-valued mapping with nonempty convex values and open lower sections, and the set $W=\{(x, y) \in X \times Y: y \in T(x)\}$ is closed in $X \times Y$. Let $f: X \times Y \times X \rightarrow 2^{Z}$ be a set-valued mapping. Assume that

(i) $M=Z \backslash(-\operatorname{int} C): X \rightarrow 2^{Z}$ is upper semicontinuous;

(ii) for each $y \in Y,-f(x, y, z)$ is $C_{x}$-0-diagonally quasiconvex;

(iii) for all $z \in X,(x, y) \mapsto-f(x, y, z)$ is upper semicontinuous on $X \times Y$ with compact values;

(iv) there exist nonempty and compact subsets $N \subseteq X, K \subseteq Y$ and nonempty, compact, and convex subsets $B \subseteq X, A \subseteq Y$ such that for all $(x, y) \in X \times Y \backslash N \times K \exists \bar{u} \in B$, $\bar{v} \in A$ satisfying $\bar{v} \in T(x)$ and $f(x, y, \bar{u}) \subseteq \operatorname{int} C(x)$.

Then, there exists $\bar{x}$ in $X$ and $\bar{y} \in T(\bar{x})$ such that $f(\bar{x}, \bar{y}, z) \nsubseteq \operatorname{int} C(\bar{x})$, for all $z \in X$. That is, the solution set of the GVEP3 is nonempty.

Corollary 3.8. Let $Z$ be a topological vector space, let $E$ and $F$ be two locally convex topological vector spaces, let $X \subseteq E$ and $Y \subseteq F$ be nonempty, closed and convex subsets, let $C: X \rightarrow 2^{Z}$ be a set-valued mapping such that $C(x)$ is a closed pointed and convex cone with $\operatorname{int} C(x) \neq \varnothing$ for each $x \in X$, let $T: X \rightarrow 2^{Y}$ be set-valued mapping with nonempty convex values and compactly open lower sections, and the set $W=\{(x, y) \in X \times Y: y \in T(x)\}$ is compactly closed in $X \times Y$. Let $f: X \times Y \times X \rightarrow 2^{Z}$ be a set-valued mapping and let $\Phi: 2^{E} \rightarrow L$ be a measure of noncompactness on E. Assume that

(i) $M=Z \backslash(-\operatorname{int} C): X \rightarrow 2^{Z}$ is upper semicontinuous on each compact subset of $X$;

(ii) for each $y \in Y,-f(x, y, z)$ is $C_{x}$-0-diagonally quasiconvex; 
(iii) for all $z \in X,(x, y) \mapsto-f(x, y, z)$ is upper semicontinuous on each compact subset of $X \times Y$ with compact values;

(iv) the set-valued map $T: X \rightarrow 2^{Y}$ is $\Phi$-condensing.

Then, there exists $\bar{x}$ in $X$ and $\bar{y} \in T(\bar{x})$ such that $f(\bar{x}, \bar{y}, z) \nsubseteq \operatorname{int} C(\bar{x})$, for all $z \in X$. That is, the solution set of the GVEP3 is nonempty.

Remark 3.9. The condition (iii) of both Corollaries 3.7 and 3.8 can be replaced by the following conditions:

(a) for all $x \in X$, for all $y \in Y, f(x, y, x) \nsubseteq \operatorname{int} C(x)$;

(b) for each $(x, y) \in X \times Y$, the set $P(x, y)=\{z \in X: f(x, y, z) \subseteq \operatorname{int} C(x)\}$ is a convex set.

If $C(x)=C$ for all $x \in X$ and $\Psi$ is replaced by a single-valued mapping $f$, then by Theorems 3.1 and 3.3, respectively, we have the following two results which are generalizations of [7, Theorems 3.2 and 3.5] and [19, Theorems 6 and 7].

Corollary 3.10. Let $Z$ be a topological vector space, let $E$ and $F$ be two Hausdorff topological vector spaces, let $X \subseteq E$ and $Y \subseteq F$ be nonempty and convex subsets, let $C \subseteq Z$ be a closed pointed and convex cone with int $C \neq \varnothing$, let $D: X \rightarrow 2^{X}$ and $T: X \rightarrow 2^{Y}$ be set-valued mappings with nonempty convex values and open lower sections, and the set $W=\{(x, y) \in X \times Y: x \in D(x)$ and $y \in T(x)\}$ is closed in $X \times Y$. Let $f: X \times Y \times X \rightarrow Z$ be a single-valued mapping. Assume that

(i) for each $y \in Y, f(x, y, z)$ is C-0-diagonally quasiconvex;

(ii) for all $z \in X,(x, y) \mapsto f(x, y, z)$ is continuous on $X \times Y$;

(iii) there exist nonempty and compact subset $N \subseteq X$ and $K \subseteq Y$ and nonempty, compact, and convex subset $B \subseteq X, A \subseteq Y$ such that for all $(x, y) \in X \times Y \backslash N \times K \exists \bar{u} \in B$, $\bar{v} \in A$ satisfying $\bar{u} \in D(x), \bar{v} \in T(x)$ and $f(x, y, \bar{u}) \in-\operatorname{int} C$.

Then, there exists $(\bar{x}, \bar{y})$ in $X \times Y$ such that

$$
\bar{x} \in D(\bar{x}), \quad \bar{y} \in T(\bar{x}) \quad f(\bar{x}, \bar{y}, z) \notin-\operatorname{int} C, \quad \forall z \in D(\bar{x}) .
$$

Corollary 3.11. Let $Z$ be a topological vector space, let $E$ and $F$ be two locally convex topological vector spaces, let $X \subseteq E$ and $Y \subseteq F$ be nonempty, closed, and convex subsets, let $C \subseteq Z$ be a closed pointed and convex cone with int $C \neq \varnothing$, let $D: X \rightarrow 2^{X}$ and $T: X \rightarrow 2^{Y}$ be set-valued mappings with nonempty convex values and compactly open lower sections, and the set $W=\{(x, y) \in X \times Y: x \in D(x)$ and $y \in T(x)\}$ is compactly closed in $X \times Y$. Let $f: X \times Y \times X \rightarrow Z$ be a single-valued mapping and let $\Phi: 2^{E} \rightarrow L$ be a measure of noncompactness on E. Assume that

(i) for each $y \in Y, f(x, y, z)$ is C-0-diagonally quasiconvex;

(ii) for all $z \in X,(x, y) \mapsto f(x, y, z)$ is continuous on each compact subset of $X \times Y$ with compact values;

(iii) the set-valued map $D \times T: X \times X \rightarrow 2^{X \times Y}$ defined as $(D \times T)(x, y)=D(x) \times T(y)$, for all $(x, y) \in X \times X$, is $\Phi$-condensing.

Then, there exists $(\bar{x}, \bar{y})$ in $X \times Y$ such that

$$
\bar{x} \in D(\bar{x}), \quad \bar{y} \in T(\bar{x}), \quad f(\bar{x}, \bar{y}, z) \notin-\operatorname{int} C, \quad \forall z \in D(\bar{x}) .
$$


Remark 3.12. The condition (ii) in both Corollaries 3.10 and 3.11 can be replaced by the following conditions:

(a) for all $x \in X$, for all $y \in Y, f(x, y, x) \notin-\operatorname{int} C$;

(b) for each $(x, y) \in X \times Y$, the set $P(x, y)=\{z \in X: f(x, y, z) \in-\operatorname{int} C\}$ is a convex set.

Let $Y=\{\bar{y}\}$. Define a set-valued mapping $T: X \rightarrow 2^{Y}$ as $T(x)=\{\bar{y}\}$ for all $x \in X$ and define another set-valued mapping $\Psi: X \times Y \times X$ as $\Psi(x, \bar{y}, z)=\varphi(x, z)$, for all $(x, \bar{y}, z) \in$ $X \times Y \times X$. Then by Theorems 3.1 and 3.3, respectively, we have following results which are generalizations of [1, Corollary 3.1].

Corollary 3.13. Let $Z$ be a topological vector space, let $E$ be a Hausdorff topological vector space, let $X \subseteq E$ be a nonempty and convex subset, let $C: X \rightarrow 2^{Z}$ be a set-valued mapping such that $C(x)$ is a closed pointed and convex cone with int $C(x) \neq \varnothing$ for each $x \in X$, let $D: X \rightarrow 2^{X}$ be a set-valued mapping with nonempty convex values and open lower sections, and the set $W=\{x \in X: x \in D(x)\}$ is closed in $X$. Let $\varphi: X \times X \rightarrow 2^{Z}$ be a set-valued mapping. Assume that

(i) $M=Z \backslash(-\operatorname{int} C): X \rightarrow 2^{Z}$ is upper semicontinuous;

(ii) $\varphi(x, z)$ is $C_{x}$-0-diagonally quasiconvex;

(iii) for all $z \in X, x \mapsto \varphi(x, z)$ is upper semicontinuous on $X$ with compact values;

(iv) there exist nonempty and compact subset $N \subseteq X$ and nonempty, compact, and convex subset $B \subseteq X$ such that for all $x \in X \backslash N \exists \bar{u} \in B$ satisfying $\bar{u} \in D(x)$ and $\varphi(x, \bar{u}) \subseteq$ $-\operatorname{int} C(x)$.

Then, there exists $\bar{x}$ in $X$ such that

$$
\bar{x} \in D(\bar{x}), \quad \varphi(\bar{x}, z) \nsubseteq-\operatorname{int} C(\bar{x}), \quad \forall z \in D(\bar{x}) .
$$

Corollary 3.14. Let $Z$ be a topological vector space, let $E$ be a locally convex topological vector space, let $X \subseteq E$ be nonempty, closed, and convex subset, let $C: X \rightarrow 2^{Z}$ be a set-valued mapping such that $C(x)$ is a closed pointed and convex cone with int $C(x) \neq \varnothing$ for each $x \in X$, let $D: X \rightarrow 2^{X}$ be set-valued mapping with nonempty convex values and compactly open lower sections, and the set $W=\{x \in X: x \in D(x)\}$ is compactly closed in $X$. Let $\varphi$ : $X \times X \rightarrow 2^{Z}$ be a set-valued mapping, and let $\Phi: 2^{E} \rightarrow L$ be a measure of noncompactness on E. Assume that

(i) $M=Z \backslash(-\operatorname{int} C): X \rightarrow 2^{Z}$ is upper semicontinuous on each compact subset of $X$;

(ii) $\varphi(x, z)$ is $C_{x}$-0-diagonally quasiconvex;

(iii) for all $z \in X, x \mapsto \varphi(x, z)$ is upper semicontinuous on each compact subset of $X$ with compact values;

(iv) the set-valued map $D: X \rightarrow 2^{X}$ is $\Phi$-condensing.

Then, there exists $\bar{x}$ in $X$ such that

$$
\bar{x} \in D(\bar{x}), \quad \varphi(\bar{x}, z) \nsubseteq-\operatorname{int} C(\bar{x}), \quad \forall z \in D(\bar{x}) .
$$

Remark 3.15. The condition (ii) in both Corollaries 3.13 and 3.14 can be replaced by the following conditions:

(a) for all $x \in X, \varphi(x, x) \nsubseteq-\operatorname{int} C(x)$;

(b) for each $x \in X$, the set $P(x)=\{z \in X: \varphi(x, z) \subseteq-\operatorname{int} C(x)\}$ is a convex set. 


\section{Acknowledgments}

This paper was supported by the National Natural Science Foundation of China (Grant no. 10171118), Education Committee Project, Research Foundation of Chongqing (Grant no. 030801), and Natural Science Foundation of Chongqing (no. 8409).

\section{References}

[1] Q. H. Ansari and F. Flores-Bazán, Generalized vector quasi-equilibrium problems with applications, Journal of Mathematical Analysis and Applications 277 (2003), no. 1, 246-256.

[2] Q. H. Ansari and J.-C. Yao, An existence result for the generalized vector equilibrium problem, Applied Mathematics Letters 12 (1999), no. 8, 53-56.

[3] J.-P. Aubin and I. Ekeland, Applied Nonlinear Analysis, Pure and Applied Mathematics (New York), John Wiley \& Sons, New York, 1984.

[4] D. Chan and J. S. Pang, The generalized quasivariational inequality problem, Mathematics of Operations Research 7 (1982), no. 2, 211-222.

[5] S.-S. Chang, B. S. Lee, X. Wu, Y. J. Cho, and G. M. Lee, On the generalized quasi-variational inequality problems, Journal of Mathematical Analysis and Applications 203 (1996), no. 3, 686711.

[6] M.-P. Chen, L.-J. Lin, and S. Park, Remarks on generalized quasi-equilibrium problems, Nonlinear Analysis 52 (2003), no. 2, 433-444.

[7] Y. Chiang, O. Chadli, and J. C. Yao, Existence of solutions to implicit vector variational inequalities, Journal of Optimization Theory and Applications 116 (2003), no. 2, 251-264.

[8] P. Cubiotti, A note on Chan and Pang's existence theorem for generalized quasi-variational inequalities, Applied Mathematics Letters 9 (1996), no. 3, 73-76.

[9] P. M. Fitzpatrick and W. V. Petryshyn, Fixed point theorems for multivalued noncompact acyclic mappings, Pacific Journal of Mathematics 54 (1974), no. 2, 17-23.

[10] J.-Y. Fu and A.-H. Wan, Generalized vector equilibrium problems with set-valued mappings, Mathematical Methods of Operations Research 56 (2002), no. 2, 259-268.

[11] W. K. Kim, Existence of maximal element and equilibrium for a nonparacompact $N$-person game, Proceedings of the American Mathematical Society 116 (1992), no. 3, 797-807.

[12] L.-J. Lin and S. Park, On some generalized quasi-equilibrium problems, Journal of Mathematical Analysis and Applications 224 (1998), no. 2, 167-181.

[13] L.-J. Lin and Z.-T. Yu, Fixed points theorems of KKM-type maps, Nonlinear Analysis 38 (1999), no. 2, 265-275.

[14] G. Mehta, K.-K. Tan, and X.-Z. Yuan, Fixed points, maximal elements and equilibria of generalized games, Nonlinear Analysis 28 (1997), no. 4, 689-699.

[15] J.-W. Peng, Generalized set-valued equilibrium problems in topological vector spaces, Journal of Chongqing Normal University 17 (2000), no. 4, 36-40 (Chinese).

[16] _ Generalized vectorial quasi-equilibrium problem on $W$-space, Journal of Mathematical Research and Exposition 22 (2002), no. 4, 519-524.

[17] C. H. Su and V. M. Sehgal, Some fixed point theorems for condensing multifunctions in locally convex spaces, Proceedings of the American Mathematical Society 50 (1975), no. 1, 150-154.

[18] G. Q. Tian and J. X. Zhou, Quasi-variational inequalities without the concavity assumption, Journal of Mathematical Analysis and Applications 172 (1993), no. 1, 289-299.

[19] X. Wu and S. K. Shen, A further generalization of Yannelis-Prabhakar's continuous selection theorem and its applications, Journal of Mathematical Analysis and Applications 197 (1996), no. 1, $61-74$.

[20] G. X.-Z. Yuan, Remarks on quasi-variational inequalities and fixed points in locally convex topological vector spaces, Applied Mathematics Letters 10 (1997), no. 6, 55-61. 
12 Generalized vector quasi-equilibrium problem

[21] X.-Z. Yuan and E. Tarafdar, Generalized quasi-variational inequalities and some applications, Nonlinear Analysis, Theory, Methods \& Applications 29 (1997), no. 1, 27-40.

[22] S. S. Zhang, Variational Inequalities and Complementarity Problem Theory with Applications, Shanghai Science and Technology, Shanghai, 1991.

[23] J. X. Zhou and G. Chen, Diagonal convexity conditions for problems in convex analysis and quasivariational inequalities, Journal of Mathematical Analysis and Applications 132 (1988), no. 1, 213-225.

Jian Wen Peng: Department of Management Science, School of Management, Fudan University, Shanghai 200433, China

Current address: College of Mathematics and Computer Science, Chongqing Normal University, Chongqing 400047, China

E-mail address: jwpeng6@yahoo.com.cn

Dao Li Zhu: Department of Management Science, School of Management, Fudan University, Shanghai 200433, China

E-mail address: d.l.zhu@fudan.edu.cn 